\title{
EQUATIONS EQUIVALENT TO NONLINEAR DIFFERENTIAL EQUATIONS
}

\author{
JOSEPH F. deSPAUTZ AND ROBERT A. LERMAN
}

1.0. Introduction. The solution of a class of ordinary nonlinear differential equations can be established in terms of the solution of a linear ordinary differential equation; this linear base equation being readily identified from the nonlinear equation. Pinney [1] found that the solution of the specific nonlinear differential equation, $y^{\prime \prime}+p(x) y^{\prime}$ $+c y^{-3}=0, c$ constant, can be expressed as $y=\left(u_{1}^{2}-u_{2}^{2}\right)^{1 / 2}$, where $u_{1}(x), u_{2}(x)$ form a fundamental set of solutions to $u^{\prime \prime}+p(x) u=0$. Thomas [2] posed the question: Which $n$th order equations have general solutions expressible in the form $y=F\left(u_{1}, \cdots, u_{n}\right)$ where $u_{1}, \cdots, u_{n}$ constitute an appropriate set of solutions of a linear equation? Thomas answered the question in terms of the first order linear base equation $u^{\prime}+p(x) u+q(x)=0$ and also in terms of the homogeneous second order equation, $u^{\prime \prime}+p(x) u^{\prime}+q(x) u=0$ where in the second order form, $F$ depends on either one variable, $u$, or $F$ is homogeneous of nonzero degree in two $u$ 's. Herbst [3] and Gergen and Dressel [4] have extended Thomas' formulation for $F$ in two variables.

The work of the previous investigators suggests the question: Which nonlinear differential equations can be solved in terms of the solutions to other, specific, nonlinear differential equations? This question is answered for the second order elliptic equation $u^{\prime \prime}=a u+b u^{3}, a, b$, constants, the results being given in Theorem 1 .

THEOREM 1. If $u$ is a solution of the elliptic equation $u^{\prime \prime}=a u+b u^{3}$ where $a, b$ are constants, then the equation $y^{\prime \prime}=f\left(y, y^{\prime}, a, b\right),\left({ }^{\prime}\right)=d / d x$, has the general solution $y=F(u)$ if and only if $f=a Z(y)+b T(y)$ $+A(y) y^{\prime 2}$ where $Z, T, A$ satisfy $\dot{Z}-A Z=1, Z \dot{T}-(3+A Z) T=0$, $(\cdot)=d / d y$. The function $F$ is found from the relationship $u^{2}=T(F) / Z(F)$.

In addition, the work of Thomas is extended to include the second order nonhomogeneous linear base equation $u^{\prime \prime}+p(x) u^{\prime}+q(x) u$ $+r(x)=0$ where the function $F$ is a function of one variable, $u$. In this extension, and also for his first order formulation, integrals of the functional relations are found.

2.0. Nonlinear equations having nonlinear bases. This section

Received by the editor May 20, 1966 and, in revised form, October 12, 1966. 
presents the derivation of the results given in Theorem 1 and some of its consequences.

If $u$ is a solution of the elliptic equation

$$
u^{\prime \prime}=a u+b u^{3}
$$

and it is assumed that the derivable nonlinear equation has the solution $y=F(u)$, then

$$
y^{\prime \prime}=F_{u}\left(a u+b u^{3}\right)+F_{u u} u^{\prime 2} .
$$

Selecting the functional relation

$$
F_{u}=g(y)
$$

with its solution in terms of $u$

$$
u=\int^{y} g(s)^{-1} d s
$$

then the equation (2-2) becomes a function of $y$ and $x$

$$
y^{\prime \prime}=a g(y) \int^{y} g(s)^{-1} d s+b g(y)\left[\int^{y} g(s)^{-1} d s\right]^{3}+g(y)^{-1} \dot{g}(y) y^{\prime 2} .
$$

Rewriting (2-4), the general second order nonlinear differential equation having (2-1) as its base equation is

$$
y^{\prime \prime}=a Z(y)+b T(y)+A(y) y^{\prime 2}
$$

where $Z, T, A$ satisfy,

$$
\dot{Z}-A Z=1, \quad Z \dot{T}-(3+A Z) T=0 .
$$

Finally, the function $F$ can be found from the integral of (2-3) as

$$
u^{2}=T(F) / Z(F) .
$$

Let $F$ be determined from (2-7) and let $y=F(u)$ be a solution of (2-5) where $Z, T, A$, satisfy (2-6). In order to show that $u$ is a solution of the elliptic equation, it is necessary to form $y^{\prime \prime}$ using (2-6) and (2-7). Once this is accomplished, it follows that $u$ satisfies (2-1).

If initial values $y_{0}, y_{0}^{\prime}$ are given, then a corresponding set of initial values $u_{0}, u_{0}^{\prime}$ can be found using $y=F(u)$. Thus $y=F(u)$ is a general solution of (2-5) and Theorem 1 is established.

An implication of Theorem 1 is that any specific solvable nonlinear equation can be used as the base equation. This suggests that the specific nonlinear base equation could be of the class having a solution in terms of solutions to linear equations. Thus the solution of the 
general nonlinear equations should be obtainable by means of a double transformation. It is easily demonstrated that this is indeed the case. However, as the general nonlinear equation does have a linear base, it will be directly solvable by the methods associated with linear base nonlinear equations. Hence, no new nonlinear equations will be generated by this double transformation.

\subsection{Nonlinear equations with linear base equations.}

3.1. First order equations. In this section it is shown that the function $F$ follows directly from Thomas' results. Thomas has shown [2, $\S 2$ ] that the general first order equation

$$
y^{\prime}+p(x) g(y)+q(x) f(y)=0
$$

where $g, f$ satisfy either

$$
f(\dot{g} / f)=1 \text { or } g(\dot{f} / g)=1 .
$$

Then the functional relation

$$
F_{u}-f(F)=0 \quad \text { or } \quad F_{u}-g(F)=0
$$

yields a solution in the form $y=F(u)$ where $u$ is a solution of the base equation

$$
u^{\prime}+p(x) u+q(x)=0 \text { or } u^{\prime}+q(x) u+p(x)=0 .
$$

However, Thomas neglected to show that $F$ can be determined directly from (3-1) and (3-2) in terms of $u$ :

$$
u=g(F) / f(F) \quad \text { or } \quad u=f(F) / g(F) .
$$

3.2 Second order equations, $F$ in one variable. In this section the results of Thomas $[2, \S 3]$ are extended to establish the solutions of the class of nonlinear equations having a linear nonhomogeneous base equation. His results are directly obtainable from those presented here.

The relations of this section form a theorem analogous to Theorem 1 and has a proof of a similar nature.

Consider the nonhomogeneous linear differential equation

$$
u^{\prime \prime}+p(x) u^{\prime}+q(x) u+r(x)=0 .
$$

If $y=F(u), y$ being the solution of the derivable nonlinear differential equation, the following second order identity can be written

$$
y^{\prime \prime}+p(x) y^{\prime}=F_{u}[-q(x) u-r(x)]+F_{u u} u^{\prime 2} .
$$

Choosing the functional relation as 


$$
F_{u}=Z(y), \quad u=\int^{y} Z(s)^{-1} d s
$$

the identity (3-4) can be written as:

$$
y^{\prime \prime}+p(x) y^{\prime}+q(x) g(y)+r(x) Z(y)=Z^{-1} Z y^{\prime 2}
$$

where $g, Z$ satisfy

$$
Z(\dot{g} / Z)=1
$$

which, using (3-6), has the integral

$$
u=g(F) / Z(F) .
$$

Let $y=F(u), F$ determined from (3-7), be a solution of (3-5) satisfying (3-6). Then $u$ satisfies the base equation (3-3).

The general form of the nonlinear equation is (3-5). If and only if $Z, g$ satisfy (3-6), then $y=F(u)$ is a general solution where $F$ is determined from (3-7) and $u$ satisfies (3-3). Finally, using (3-6) and the coefficient of $y^{\prime 2}$, an equivalence can be established with Thomas' results [2, equations (3.1), (3.2)] with the added integral of the functional relation.

\section{REFERENCES}

1. E. Pinney, The nonlinear differential equation $y^{\prime \prime}+p(x) y+c y^{-3}=0$, Proc. Amer. Math. Soc. 1 (1950), 581.

2. J. M. Thomas, Equations equivalent to a linear differential equation, Proc. Amer. Math. Soc. 3 (1952), 899-903.

3. R. T. Herbst, The equivalence of linear and nonlinear differential equations, Proc. Amer. Math. Soc. 7 (1956), 95-97.

4. J. J. Gergen and F. G. Dressel, Second order linear and nonlinear differential equations, Proc. Amer. Math. Soc. 4 (1965), 767-773.

Hamilton Standard, Windsor Locks, Connecticut 